\title{
INFLUENZAL MENINGITIS
}

\author{
WITH REPORT OF A CASE *
}

ISAAC A. ABT, M.D., AND I. HARRISON TUMPEER, S.M., M.D.

The literature contains many observations on meningitis caused by an organism resembling the influenza bacillus. The relationship of this organism to the so-called true influenza bacillus of Pfeiffer is still a matter of controversy. We wish to report such a case of meningitis and describe the results of the study of the organism. A variety of interesting laboratory findings were made, but the picture of the purulent meningitis remained clear cut. The bacillus was isolated from the spinal fluid, blood, throat, nose and nasopharynx. Cultures were studied and animals inoculated. The spinal fluid yielded a four plus Wassermann and reduced colloidal gold suspensions in the syphilitic zone. Morphologically typical diphtheria bacilli were found in large numbers in a nasal culture. Death occurred ten days after the onset. A necropsy was not permitted.

\section{REPORT CF CASE}

History.-The patient was referred to one of us (I. A. A.) by Dr. Mary Shutan. The child was a white male, aged 17 months, whose symptoms became manifest three days before admission to the hospital. On the first day of the illness the temperature rose to $102.4 \mathrm{~F}$., but the child played and seemed to be fairly well. The following day the temperature dropped after a warm bath, but the child was very restless and vomited after nearly every ingestion of solids or liquids. There was no vomiting the next day, but occasionally afterward. Besides crying and being restless, muscular twitchings were noticed the second day, but convulsions did not occur. The right drum membrane was found bulging, and paracentesis yielded thick pus. This was repeated on both ears before admission to the hospital, and each time pus was obtained. The temperature had been subnormal for more than a day before admission, but rose subsequently. The child became very cold; rigidity was present, and the mouth had to be forced open to insert food. Strabismus was first noticed on the third day. There was no urination for periods of twelve hours on two occasions.

The birth and feeding histories were not remarkable. The patient was the third child. The parents and two other children were apparently well, and there was no history of miscarriages. The child had scarlet fever four months previously.

Physical Examination.-The patient was well nourished. He was restless and appeared to be very ill. The veins of the face were somewhat dilated. The right drum membrane was red, and there was a purulent discharge in the canal. Cultures contained staphylococci but no influenza bacilli. The left drum

* Received for publication, Dec. 20, 1920.

* From the Sarah Morris Memorial Hospital. 
was bulging and cloudy. There was an internal strabismus of the left eye; no ptosis or nystagmus. The pupils were equal and reacted to light. There was marked rigidity of the neck and extremities, and the spinal column was flexed with difficulty. The Brudzinski sign was positive, but the Kernig sign was negative. The reflexes were all very active, but no pathologic reflexes were noted. The chest and abdomen were not abnormal.

Laboratory Findings.-Blood examination on admission showed 5,120,000 red cells and 7,600 leukocytes, of which 72 per cent. were polymorphonuclears, 28 per cent. small mononuclears, 3 per cent. large mononuclears and 1 per cent. transitionals.

A morning specimen of urine was cloudy, specific gravity of 1.020 ; acid, and contained albumin but no sugar or acetone. There were many granular casts, a few leukocytes and occasional erythrocytes.

Throat cultures were twice reported free from diphtheria bacilli.

Spinal puncture on admission yielded 40 c.c. of turbid fluid under markedly increased pressure. A pellicle formed in five minutes. There were 3,500 cells

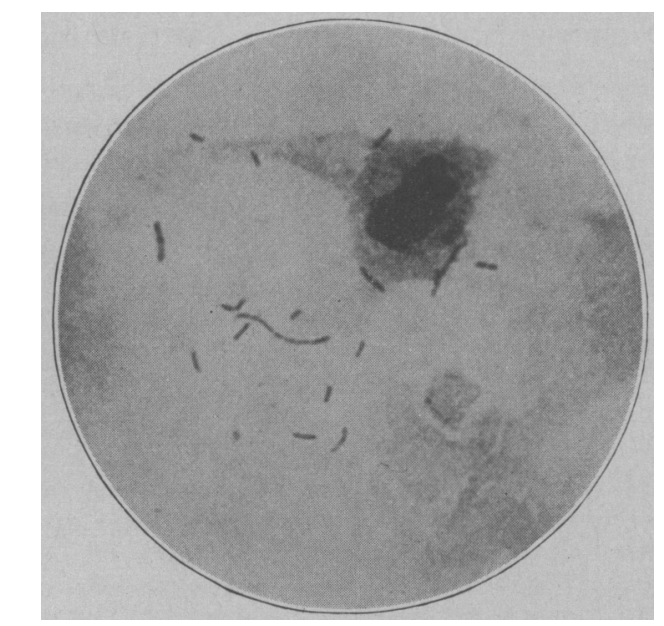

Direct smear of the spinal fluid sediment showing pleomorphism of the organism. The long thread form appears to be a series of influenza bacilli joined end to end by their cell membranes. Note the appearance of polar staining (methylene blue stain).

of which 84 per cent. were polymorphonuclears, 9 per cent. lymphocytes and 7 per cent. endothelial cells. The Noguchi, Ross-Jones and Noinne tests were markedly positive. The Wassermann test was reported ++++ , and the colloidal gold test showed a reduction in the syphilitic zone.

Direct smears of the pellicle showed many polymorphonuclear cells and a gram-negative, bizarre, pleomorphic bacillus, some forms of which were long threads, others plump and tiny. Cultures yielded the same organism. Its further history is given below.

Subsequent Course-The day following admission 15 c.c. spinal fluid was removed. This specimen was more turbid than the first, contained 10,000 cells and the same organism. At this time, ethyl hydrpcuprein, 2 gr., dissolved in 8 c.c. of water was injected into the spinal canal.

On the third day since admission, the sixth of the illness, the temperature rose to $105.4 \mathrm{~F}$. Despite this the child seemed bright, but was very restless. There was no Kernig sign, but the Brudzinski was slightly indicated. The 
refiexes were markedly exaggerated, and the strabismus of the left eye was unchanged.

On the fifth day, the eighth of the illness, the temperature was $103.6 \mathrm{~F}$. The child was comatose, and the Kernig and Brudzinski signs were now quite marked.

On the day before death, 10,000 units of diphtheria antitoxin was injected deep intramuscularly because of the finding of diphtheria bacilli in the nose the day before, despite the absence of clinical symptoms.

Attempts to obtain spinal fluid at this time failed although it was apparent that the needle was in the canal. It was concluded that the exudate had become too thick to escape through the needle inasmuch as postmortem examinations of influenzal meningitis reveal a thick, plastered exudate over the cord and brain.

In the course of the disease, at the hospital, the temperature varied between 103.8 and $105.8 \mathrm{~F}$. The pulse varied from 135 to 170 , and respirations from 32 to 60 .

Bacteriologic Examination. - A blood culture made on the day preceding death contained influenza bacilli. Two dilutions were made in preparing the culture. One was contaminated with staphylococci. A positive result was obtained even in the dilution of 0.5 c.c. of blood to 20 c.c. of plain broth. The same pleomorphism was noted as in the spinal fluid. When subculture was made on chocolate agar, typical influenza bacilli were found, with an occasional long form.

Smears on blood agar yielded colonies of influenza bacilli from the nose, throat and posterior nasopharyngeal wall. A culture from the nose on Loeffler's medium yielded morphologically typical diphtheria bacilli.

A cultural study was made of the organism obtained from the spinal fluid. Direct smears contained slender, gram-negative bacilli of different lengths and widths. Culture on blood agar plates yielded fine, dew drop colonies with pleomorphic, gram-negative bacilli. No growth was obtained from the same fluid on plain agar or plain broth. Subcultures from the blood agar plate on chocolate agar yielded typical influenza bacilli, but a few thread forms were present. On Avery's medium the pleomorphism was more marked, and smears resembled the direct smears obtained from the original fluid. There was no growth on subculture on plain agar, plain broth, glucose broth and serum inulin water.

Animals were inoculated without producing death. A rabbit was given an intravenous injection of a twenty-four hour culture suspended in salt solution. Death did not occur although several subsequent injections were made. A white mouse was injected intraperitoneally. Instillation of a twenty-four hour growth into the nasal cavity of a guinea-pig to determine whether infection would occur by that route produced no effect.

\section{DISCUSSION}

Cellular Content of the Spinal Fluid.-The cell count of the spinal fluid in influenzal meningitis varies, although most observers report the predominance of polymorphonuclear cells. In our case we found 3,400 and 10,000 cells, most of which were polymorphonuclear. Hill's ${ }^{1}$ patient had fifty cells, mainly lymphocytes. Davis ${ }^{2}$ reported four cases in which turbid fluid was present under high pressure, the polymorphonuclear cells forming from 6 to 80 per cent. of the total count. He

1. Hill: New York M. J. 107:345, 1918.

2. Davis: Am. J. Dis. Child. 1:249 (Feb.) 1911. 
remarks on the presence of endothelial cells, which we have also found. Boland ${ }^{3}$ reports 1,000 cells with 90 per cent. polymorphonuclear cells. The fluid in this case became so thick that it finally failed to pass through the needle. Brown ${ }^{4}$ describes the case of a 5 months old infant who had three punctures on successive days. There were 1,600 cells with 96 per cent. polymorphonuclear cells and 3,400 and 3,600 cells the two following days, all of which were polymorphonuclears. $\mathrm{He}$ reports another case, an infant, 1 year old, with 6,080 cells, 73 per cent. of which were polymorphonuclears. Ross and Moore's ${ }^{5}$ case had 86 per cent. polymorphonuclears, and Moody's ${ }^{B} 11$ months old patient had 256 cells, of which 230 were polymorphonuclears. Another patient, aged 8 months, had 490 cells with 90 per cent. polymorphonuclears. Bhat ${ }^{7}$ had a 14 months old infant with 150 cells, of which 54 per cent. were polymorphonuclears and 46 per cent. lymphocytes. There were eighteen endothelial cells for 100 leukocytes. Later punctures showed more polymorphonuclear cells. Stone ${ }^{8}$ collects two cases, Hills and his own, which had bloody fluid uniformly.

Blood Count.-Few reports in the literature mention blood counts. In our case there were 7,600 cells of which 72 per cent. were polymorphonuclears, 28 per cent. small mononuclears, 3 per cent. large mononuclears and 1 per cent. transitionals. A normal infant of 17 months averages about 40 per cent. polymorphonuclears and 60 per cent. mononuclears. There appears to be a slight leukopenia in our case although the cell count of the spinal fluid is high and the majority of the cells are polymorphontclears. The further significance of the leukopenia in influenza is a nonspecific activity of the leukocytes. as demonstrated by Tunnicliff. ${ }^{9}$ She states that this decreased activity continues until the patient recovers, in which case the leukocytes become normally active and increase in number. She suggests that leukopenia and diminished phagocytosis may account for the severity and frequency of secondary infections. Hill and Packard ${ }^{10}$ state that the blood shows a moderate leukocytosis, usually under 15,000, with an increase in polymorphonuclear cells. Stone had a case in a girl, 15 years old, who had 83 per cent. polymorphonuclear cells. Moody's second patient had 12,300 leukocytes.

3. Boland: Lancet 2:704, 1915.

4. Brown: Canad. M. A. J. 5:1076, 1915.

5. Ross and Moore: Brit. M. J. 2:1056, 1913.

6. Moody: J. Missouri M. A. 13:328, 1916.

7. Bhat: Lancet 2:384, 1917.

8. Stone: Texas State J. M. 15:318, 1920.

9. Tunnicliff: J. A. M. A. 71:1733 (Nov. 23) 1918.

10. Hill and Packard: Quoted in Editorial, Lancet 2:189, 1915. 
The Organism in the Spinal Fluid.-The organism found in the spinal fluid has been abundantly described. Pleomorphism in culture and smears from the meninges is its most striking feature. The descriptions are much alike. The bacilli are gram-negative and grow only on blood medium as dew drop transparent colonies. They vary in size from small, plump, coccoid organisms to long thread forms resembling leptothrix with which they have often been confused. Henry ${ }^{11}$ states that influenza-like organisms in some cases exhibit bipolar staining which makes them resemble diplococci. $\mathrm{He}$ also remarks that sometimes the long forms do not appear for three or four days, while in other cases they appear early. The longer forms often predominate, and the organisms are practically always extracellular. This is probably an indication of diminished phagocytosis. The pleomorphism persists in subcultures on blood agar. Studying the development of pleomorphism, Brown stated that after from fifteen to twenty-four hours, minute regular bacilli appeared, among which threads were seen. On the second day, the curved forms and threads were usually increased, and the bacilli were thicker. Wollstein ${ }^{12}$ describes the influenza bacillus as being a slender rod, varying in size, staining deeply at the poles and gram-negative. She emphasizes that the invariable and most prominent characters are, first, hemophiiic property and second, pleomorphism. In one of her cases she found long forms with curved ends, often clubbed, corresponding to involution forms found in old cultures and a few characteristic small forms. In two others there were long threads but the typical small forms predominated. Bhat also speaks of long forms which were found end to end by cell membrane and Ager and Avery ${ }^{13}$ remarked that the organism from their case exhibited polar staining and varied in size, often occurring in threadlike forms. It has been stated that the chains and convolutions are bacilli placed end to end united by continuation of the cell membrane.

The organism from Moody's second case did not grow on Loeffler's medium.

Ross and Moore isolated the organism from the fluid of the right ventricle.

Following Jordan's ${ }^{14}$ observation on indol formation by the influenza bacillus, Rivers ${ }^{15}$ found that eleven of twelve cases of influenzal meningitis yielded an indol reaction on the spinal fluid directly.

11. Henry: J. Path. \& Bacteriol. 17:174, 1912.

12. Wollstein: Am. J. Dis. Child. 1:42 (Jan.) 1911.

13. Ager and Avery: Arch. Pediat. 27:284, 1910.

14. Jordan: J. A. M. A. 72:1542 (May 24) 1919.

15. Rivers: J. A. M. A. 75:1495 (Nov. 27) 1920. 
Its Relation to Pfeiffer's Bacillus.-The pleomorphism of the organism in this type of meningitis raises the question of its classification. Invariably, it is hemoglobinophilic. Its growth is always in the form of dew drop colonies only produced by the influenza bacillus described by Pfeiffer. It is always gram-negative. However, animal inoculations give varying results, and its morphology is somewhat perplexing. There are many who believe that the organism producing meningitis is a bacillus quite distinct from Pfeiffer's bacillus. They insist that this is a pseudo-influenza or an influenzoid organism. Cohen ${ }^{16}$ is the chief proponent of this separate organism theory. He states that a septicemic type of influenza bacillus exists which causes meningitis, inasmuch as experimental septicemia cannot be produced, except in those with influenzal meningitis. He concurs with Pfeiffer's original view that the organism does not occur in the blood, but that the meningeal type produces a septicemia in rabbits with serous lesions, as in the human. He has found that vaccination may be carried out against the organism, but the serum of vaccinated animals is neither curative nor preventive, and phagocytic and agglutinative reactions do not distinguish this organism sufficiently from Pfeiffer's bacillus. The differences are summed up in this way by Henry. The organism from meningitis shows bacillary and filamentous forms in culture more readily than does Pfeiffer's organism. It is more readily recovered from heart's blood and tissues than the organism from influenza. It tends to kill laboratory animals by septicemia rather than by toxemia, and fatal results occur with much greater regularity than is the case with Pfeiffer's bacillus.

On the other hand, Wollstein believes that it may be considered as conclusively established that a pseudo-influenza bacillus producing pathologic conditions in human beings as distinct from the true influenza bacillus does not exist. She suggests that the frequent finding of the influenza bacillus in endocarditis, purulent arthritis, empyema, appendicitis, peritonitis, meningitis, otitis, as well as its frequent occurrence in the bronchial and nasopharyngeal secretions in cases of clinical influenza indicates that that organism, like the pneumococcus, is capable of causing inflammations of the serous membranes anywhere in the body. Along these lines Rivers offers the suggestion that the influenza ${ }^{17}$ bacillus may be a group of organisms like the streptococcus, which has been divided into hemolytic and nonhemolytic strains and further subdivided by cultural characteristics and serologic tests. He exacts ten requirements for the true influenza bacillus. These tests are hemophilic quality, colony formation, hemolytic test, reaction to Gram's

16. Cohen: Ann. de 1'Inst. Past. 23:273, 1909.

17. Rivers: Bull. Johns Hopkins Hosp. 31:50, 1920. 
stain, morphology, motility, indol formation, found by Jordan to occur in ten of thirteen strains, reduction of nitrates to nitrites, amylase formation and reaction in blood, broth and milk. Further evidence that the meningeal organism is the true influenza bacillus is offered by Slawyk, ${ }^{18}$ who isolated a bacillus from the blood and spinal fluid in meningitis which Pfeiffer agreed was the influenza bacillus, and by Thursfield, ${ }^{19}$ who found the organism in the blood without meningitis. Flexner ${ }^{20}$ interprets the situation by expressing that nearly all cases of influenzal meningitis are examples of bacteremia, since the bacilli are frequently cultivated in large numbers from the blood during life or at necropsy.

To compromise the two views, Henry states that there is a group of hemoglobinophilic organisms causing meningitis whose members range from the influenza bacillus of Pfeiffer which, when it kills, does so by toxemia, to Cohen's organism of very high virulence which kills laboratory animals regularly by septicemia. Ross and Moore state it is impossible to dogmatize whether the organism isolated from sporadic cases of meningitis is identical with the organism in the respiratory tract in influenza. It is sufficient to say that there is a group of cases showing pyemic characters in which meningitis is a common occurrence and in which the only organism present is a hemophilic bacillus having the characteristics of the influenza bacillus. After all these reports and opinions, is it not possible that the influenza bacillus is subject to involutions? The conditions of growth in the spinal canal are somewhat comparable to the conditions in a fluid medium such as Avery's medium. We found that the typical influenza bacilli which were grown by subculture of the pleomorphic bacillus from the spinal fluid on the solid medium of chocolate agar reverted to pleomorphism when grown in Avery's medium.

Animal Inoculation.-The organism in our case was not pathogenic for guinea-pigs, rabbits or mice, despite repeated injection. There are reports of other organisms which illustrate the low pathogenicity we found. Henry injected three rabbits intravenously, intraperitoneally and subcutaneously, respectively, with no illness resulting and no findings when the animals were killed. Ager and Avery failed to produce infection in a guinea-pig inoculated with the organism from their patients, but a rabbit previously bled 15 e.c. died in forty-eight hours. Torrey's ${ }^{21}$ organism was of low pathogenicity tested by rabbits. Ritchie's ${ }^{22}$ observations differ to this extent. The real influenza bacillus

18. Slawyk: Ztschr. f. Hyg. 32:443, 1899.

19. Thursfield: Quoted by Rivers, Reference 17.

20. Flexner: J. A. M. A. 57:16 (July 3) 1911.

21. Torrey: Am. J. M. Sc. 152:403, 1915.

22. Ritchie: J. Path. \& Bacteriol. 14:615, 1910. 
is at first innocuous to guinea-pigs but after subculture for five months it gives a septicemia in guinea-pigs by intraperitoneal injection.

Many observers obtain positive results by animal inoculation. The second generation of the organism from Hill's case inoculated intraperitoneally into a white mouse caused death in less than twenty-four hours. Intravenous injection into a $900 \mathrm{gm}$. rabbit was followed by death in thirty-six hours, and the organism was recovered from the heart's blood. Wollstein's work in 1911 demonstrated that mice were highly susceptible to small injections intraperitoneally whether the cerebrospinal fluid or pure cultures of the organism were used. Animal passage through mice did not appreciably increase the virulence, although only one half the dose was required to kill after passage.

Guinea-pigs died in from twelve to twenty-six hours after injection of from one-half to whole cultures. Rarely was phagocytosis found. although there were polymorphonuclear cells in the peritoneum. Sometimes, the animals lived for three or four days.

Rabbits injected with twenty-four hour cultures died in from fifteen to thirty-six hours, and the bacilli were recovered from the mucosal surface of the upper nasal cavities.

Monkeys injected subdurally by spinal puncture yielded symptoms in from six to twelve hours.

Wollstein found that out of a large number of strains, four were found to be virulent for rabbits. Three of these were obtained from influenzal meningitis spinal fluid. Many necropsies were examined for bacilli from the blood without results. She states that the bacilli are more frequently isolated from the blood postmortem in the cases of meningitis. All strains were found virulent for small animals. In general, the high virulence for human beings ran parallel with the effects on rabbits and monkeys.

Albert and Kelman ${ }^{23}$ find that the bacilli are usually destroyed rapidly after their introduction into the body, but following intraperitoneal injections, the organism may appear in the blood. They also state that the bacillus produces a toxin which is fatal to mice, guineapigs and rabbits almost as soon as broth cultures. Death occurs in from one and one-half hours to thirty days after injection. They suggest that death occurring after four days may be caused by secondary invading organisms. It is their experience that guinea-pigs are more uniformly susceptible than rabbits or mice, and rabbits show a greater individual variation in susceptibility. On the basis of their work, they conclude that the influenza bacillus is distinctly pathogenic to mice, guinea-pigs and rabbits, but that this quality is apparently limited to certain cultures or strains of the organism.

23. Albert and Kelman: J. Infect. Dis. 25:433, 1919. 


\section{PATHOGENESIS}

The influenza bacillus behaves like the pneumococcus. It has been found in the mouth in 43 per cent. of persons ${ }^{24}$ examined. Considering the upper respiratory tract as the most frequent portal of entry, Wollstein explains the localization of the organism in the middle ear, bronchi and lungs. In Brown's case of a 5 months' old infant there was double otitis media with preceding bronchitis, in which the influenza bacillus was found in pure culture. The blood, nasopharyngeal and postmortem culture of brain, heart's blood, lung and mastoid all yielded the organism. This well illustrates the belief of Wollstein that the influenza bacillus acts like the pneumococcus and is equally as capable of producing inflammations of the serous membranes anywhere in the body.

To determine the rôle of the upper respiratory tract in the etiology of influenzal infections Bloomfield ${ }^{25}$ introduced the organism into the upper respiratory passages. Several strains so introduced disappeared in from one to three days. No carrier states were produced, and no local or general pathologic processes resulted from the inoculation. Bacilli isolated more than twenty-four hours after introduction were shown to be different strains, and they were not viable after suspension in saliva for twenty-four hours at 37 degrees $C$.

The middle ear undoubtedly plays an important rôle in the development of influenzal meningitis in children. Ritchie quotes Koṣsel as finding in 108 postmortem examinations in children under 1 year of age, eighty-five cases of otitis media, over half of which were of influenzal meningitis. Hartman and Honel ${ }^{26}$ have found 90 per cent. of cases of otitis media in infants of influenzal origin. Our case may have been one of influenzal otitis media originally although late cultures showed staphylococci which may have been secondary invaders. Suppuration of the frontal and maxillary sinuses was the source of infection in Torrey's first case. Henry states that infection may come directly through the carious bone or by blood vessels running through the remains of the petrosal squamosal suture from the antrum to the middle fossa without direct extension or caries. The nasopharynx, he suggests, may be the portal through which the bacilli are conveyed by the nasal lymphatics to the anterior fossa of the skull, through the cribriform plate of the ethmoid.

Opinion seems to favor a bacteriemia as the mechanism in the production of meningitis, although this cannot always be demonstrated. Inoculation of the bacilli intravenously in rabbits has resulted in positive blood culture postmortem. Following intraperitoneal injection they

24. Pritchell and Stillman: J. Exper. M. 29:259, 1919.

25. Bloomfield: Bull. Johns Hopkins Hosp. 31:85, 1920.

26. Hartman and Honel: Quoted by Henry, Reference 11. 
may appear in the blood, probably depending on the virulence of the organism and the lowered resistance on the part of the body. ${ }^{23}$ After subdural inoculation, they may be recovered from the upper nasal mucosa, suggesting that they are excreted at this point. ${ }^{13}$ Since they also reach the blood, they may be secreted from the blood and not directly through the meninges. After intravenous injection of influenza bacilli into rabbits they are found in the secretions of the mucosa. They are excreted by the kidneys in rabbits and guinea-pigs, and pure cultures may be obtained from the surface of the cord and brain in rabbits and guinea-pigs inoculated intravenously or intraperitoneally, although no lesions or inflammations of the meninges are found. From this experimental evidence, Wollstein believes that the bacilli may be present in the spinal fluid without causing meningitis and permitting a clear fluid.

In furtherance of the theory of bacteremic pathogenesis of influenzal meningitis, Henry thinks that the occurrence of bronchopneumonia, pleurisy and pericardial inflammations, complicated with meningitis, allows the assumption that the bacilli are carried to the meninges by the blood stream or pass to the cervical meninges by the thoracic lymphatics, as in some cases of tuberculous meningitis. It will be remembered that Flexner stated that nearly all cases of influenzal meningitis are examples of bacteriemia, as demonstrated by the frequent positive blood findings during life or at necropsy. Davis states that the meningeal infection is probably hematogenous in origin, even though the heart's blood cultures after death may be negative. Difficulties encountered in making blood cultures in young children during life are responsible for the absence of data on this point, but we can say that in the majority of the fatal cases of influenzal meningitis a general blood infection occurs whether this invasion is primary or secondary.

\section{PROGNOSIS}

More cases of influenzal meningitis occur in infants and young children than in adults, and the process is more severe in children. Stone collected 105 cases, with a mortality of 91.4 per cent., nine patients recovering.

\section{TREATMENT}

To reduce the high mortality many agencies have been tried. From the medicinal standpoint, hexamethylenetetramin, in 10 grains doses, at four hour intervals was followed by improvement in one case reported by Ross and Moore although the final outcome is not reported.

The diminished phagocytosis in influenzal infections suggests that favorable results may be obtained by measures aimed to increase leukocytic activity. Practically no phagocytosis occurs, as is evidenced by 
the extracellular arrangement of the organism in the spinal fluid. In fact, there seems to be evidence for Torrey's contention that protective substances may pass out into the spinal fluid following spinal puncture due to lowered pressure and consequent increased outpouring into the cerebrospinal space. Following lumbar puncture, there is increased phagocytosis and decrease in the organism. In one of Torrey's' cases, the organism became intracellular at the second puncture. The good effect following tapping, he states, may also be due to relief of high pressure, improved blood supply, and removal of toxins.

Spinal lavage in Ross and Moore's case resulted in a less involved spinal cord than brain.

Regarding specific therapy, Cohen states that animals may be vaccinated against the organism, but that the serum of vaccinated animals is neither curative nor preventive. Tunnicliff suggests that convalescent serum or immune horse serum may promote leukocytosis and increase antibody content of the serum. In this connection Johnson ${ }^{27}$ reports the case of a 3 year old boy who recovered following the injection of convalescent serum intraspinally and subcutaneously. It is to be expected that normal serum may increase phagocytosis and improve the condition.

\section{CONCLUSIONS}

1. Influenzal meningitis is accompanied by a widespread distribution of the influenza bacillus in the upper respiratory tract.

2. The organism is easily cultivated from the spinal fluid and in many cases from the blood.

3. The organism from the spinal fluid is usually pleomorphic, showing many long thread forms.

4. It seems plausible that the pleomorphism is but a minor variation from the so-called typical influenza bacillus, and that this may be due to the peculiar conditions of its habitat.

5. Spinal fluid usually contains a large number of polymorphonuclear cells which show no tendency to phagocytosis.

6. The blood count does not give the typical leukopenia of respiratory influenza.

7. Animals show a variable response to injections of the organism, although the bacillus is usually pathogenic for laboratory animals.

8. Prognosis of influenzal meningitis is very grave.

9. The most rational treatment at the present time is the administration of convalescent or normal serum and frequent removal of spinal fluid. The serum contains antibodies and promotes phagocytosis, and spinal puncture removes fluid and increases phagocytosis.

27. Johnson: Arch. Pediat. 36:82, 1919. 